\title{
Experiments with Cooperative Networked Control of Underwater Robots
}

\author{
Matthew Dunbabin* Iuliu Vasilescu ${ }^{\dagger}$ \\ Peter Corke ${ }^{\dagger}$ Daniela Rus*
}

\begin{abstract}
We present details and results obtained with an underwater system comprising two different autonomous underwater robots (AUV) and ten static underwater nodes (USN) networked together optically and acoustically. The AUVs can locate and hover above the static nodes for data upload, and they can perform network maintenance functions such as deployment, relocation, and recovery. The AUVs can also locate each other, dock, and move using coordinated control that takes advantage of each AUV's strength.
\end{abstract}

\section{Introduction}

We are developing small autonomous underwater robots that are cooperative, adaptive, and can establish ad-hoc underwater networks. Such robots will permit the exploration and monitoring of underwater environments, allowing applications such as long-term monitoring of underwater habitats, monitoring and surveillance of ports, modeling the impact of weather and ground activities (such as manufacturing and agriculture) on the water quality, and underwater geochemical prospecting. Each of these applications requires long term underwater presence over a large area and adaptation to triggers in the environment, by positioning and repositioning the robot or adjusting the sampling rate.

\footnotetext{
${ }^{*}$ CSIRO ICT Centre, Brisbane 4069, Australia. Email first. last@csiro.au

${ }^{\dagger}$ MIT Computer Science and Artificial Intelligence Laboratory, Cambridge MA 02493, USA. Email \{iuliuv, rus\}@csail.mit.edu
} 
Cooperation is an important aspect in designing useful underwater robots. Underwater robots that are working collaboratively are capable of achieving tasks beyond that of individual robots. Longrange and long-duration underwater operations require significant power and therefore a large robot. Once at the destination the large size may not be advantageous if the robot has to perform many maneuvers: the larger the robot, the greater the power required. Furthermore, large robots are less maneuverable than small robots. When the tasks at the robot destination involve intricate trajectories or traversal of narrow tunnels (for purposes such as monitoring, exploration, shipwreck inspection, imaging, or data muling from an already deployed sensor network) a smaller robot is more useful. We need robot systems that meet both needs: (1) can sustain long-range travel and (2) are maneuverable at the destination.

We have developed a heterogeneous cooperating team of underwater robots and underwater sensor network nodes to address these kinds of applications. The teams cooperate by interacting with each other either as coupled systems (e.g., our two robots dock together and cooperate to travel together) or as networked systems (e.g., the robots deploy an underwater sensor network that form a navigation support system for location, positioning, tracking, and guidance.)

In this paper we describe our work in on control for cooperation with underwater robots. Specifically, we describe two underwater robots developed at CSIRO and MIT respectively, and a means of networking them acoustically and optically. We also describe algorithms that enable the autonomous robots to dock underwater, to travel together to a destination and to separate for individual work. The cooperation control algorithms enable the robots to pool their resources for more effective navigation (e.g., the coupled robot system is overactuated, whereas the two individual robots are underactuated). Here we focus on two skill that enable cooperative navigation: (1) docking between two moving robots and (2) cooperative control and describe our algorithms and experiments. This work builds on exciting new directions in networked underwater robotics $[1,4,7]$. In our previous work we described the mobile sensor network infrastructure [6] and a data muling algorithm and experiments [3]. 

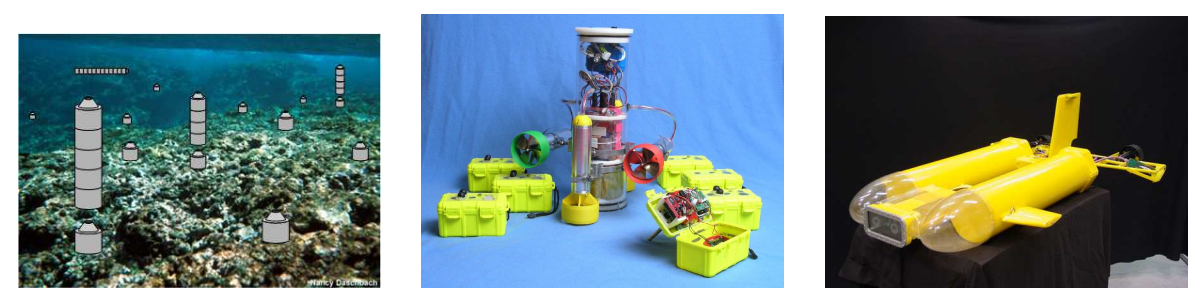

Figure 1: Autonomous modular underwater vehicles deploying and collecting a sensor network. The proposed sensor modules stack up at the bottom of the underwater vehicle. They do not move on their own. One or more autonomous vehicles deploy the sensor modules by releasing them at desired locations. To collect a sensor, an autonomous vehicle docks with the sensor and connects it to its body. The center figure shows the AMOUR underwater robot and the Aquafleck underwater sensor nodes. The right figure shows the Starbug underwater robot.

\section{Technical Approach}

\subsection{System Description}

Our system consists of underwater sensor network nodes called Aquafleck and two autonomous robots called Starbug and Amour. Figure 1 shows the cooperation concept and the two autonomous robot used in this work. The hardware and networking infrastructure of this system has been described in detail in [6]. Aquaflecks are underwater sensor network nodes that package together computation, acoustic and optical communication, and a suite of sensors in a water-tight enclosure. A low-cost/low-power acoustic communication module using $30 \mathrm{kHz}$ FSK/PPM modulation with a range of $200 \mathrm{~m}$ omnidirectional, a data rate of 100bit/s and is also used for ranging. An optical communications board drives a high-power LED and a sensitive matched photodiode [6]. The LED has a cone of 30 degrees and a maximum data rate of $320 \mathrm{kbits} / \mathrm{s}$. Each node has a pressure sensor, temperature sensor, and a CMUCam camera. The top of the box contains a $170 \mathrm{~mm}$ rod with an LED beacon, which an AUV can use to locate the box, dock, and pick it up.

Amour is an AUV developed at MIT. It has on-board computation, storage, batteries, and acoustic and optical communication. Its key performance specifications are: mass $(11 \mathrm{~kg})$, length $43.3 \mathrm{~cm}$, 
diameter $15.3 \mathrm{~cm}$, maximum forward thrust 70N, maximum linear speed $1 \mathrm{~m} / \mathrm{s}$, maximum rotation speed $360 \mathrm{deg} / \mathrm{s}$, and endurance 10 hours using lithium power battery. It has four external thrusters with a maximum power of $150 \mathrm{~W}$ and a maximum static thrust of 35 $\mathrm{N}$ each. Two thrusters act vertically and two thrusters are act horizontally to provide forward-backward propulsion and yaw control. The bottom cap of the robot has a cone shaped cavity, designed for maximum mechanical reliability in docking and for optical communication.

Starbug is a hybrid AUV developed at CSIRO [2]. It has a powerful onboard vision system functionality. Its key performance specifications are: mass $26 \mathrm{~kg}$, length $1.2 \mathrm{~m}$ (folding to $0.8 \mathrm{~m}$ for transport), maximum forward thrust $20 \mathrm{~N}$, maximum speed $1.5 \mathrm{~m} / \mathrm{s}$, and maximum endurance of 3.5 hours $(8 \mathrm{~km}$ at $0.7 \mathrm{~m} / \mathrm{s})$ with current lead-acid battery technology. The vehicle is fully actuated with six thrusters providing forward, lateral and vertical translations as well as yaw, roll and pitch rotations. Starbug has two stereo vision heads. One looking downward for sea-floor altitude and speed estimation as well as mapping, and the other looking forward for obstacle avoidance.

\subsection{Docking}

A critical part of this system is the ability of a vehicle to locate and dock with the other system. We approached this problem by using the Acquafleck underwater sensor network nodes as the docking interface (see [6]). We developed algorithms that enable each of the two robots to take advantage of their resources. Starbug uses vision to locate the bright yellow Aquaflecks. Amour relies on the optical beacon on the Aquafleck as well as its ability to communicate optically and acoustically to guide itself to dock with the node. Amour docks with Starbug in the same way, by using an Aquafleck node integrated in the body of Starbug.

\subsection{Cooperative Modeling and Control}

Once docked, two AUV have the ability travel together combining their resources and ensuring that they reach the same destination at the same time. We have developed a cooperative control algorithm that enables Starbug and Amour to maneuver and travel together.

The objectives of this work was to investigate (1) when an AUV is docked to another AUV with an optical communication link between 
the two, can one control the other to obtain cooperative motion, and (2) what happens to the dynamics of the system when two indendent systems are combined.

In this investigation, Starbug was fitted with a modified Aquafleck which allowed Amour to physically lock onto Starbug and transfer forces from its thrusters to Starbug in the $x, y$ and $z$ directions, including moments about $x$ and $y$, whilst free to spin about the $z$-axis as shown in Figure 2(b). The docking Aquafleck optically passed command signals from Starbug to Amour.

Generating accurate dynamic models of an AUV is generally a difficult task requiring significant experimental evaluation to determine hydrodynamic coefficients. Empirical results for standard AUV configurations can reduce the amount of experimentation, however, both Starbug and Amour are not standard. For this application the models need only provide a gross estimate of the combined vehicle motion, not high fidelity motion. Therefore, models for both AUVs were developed individually based on the simplified dynamics approach provided by [5]. As both AUV's have few experimentally determined hydrodynamic coefficients, key parameters are estimated using a series of cylinders and flat plates to represent the vehicle and superimposing the hydrodynamics effects. This assumption typically leads to the greatest source of modelling error.

The two models include estimates of drag, lift and added mass, as well as weight, buoyancy, thrust and reaction torques. These models were individually tuned to approximate vehicle performance measured and observed during experimentation. The combination of these two models causes some added problems due to the change in mass, inertia, and the center of gravity $(\operatorname{cog})$ and buoyancy (cob) locations. Therefore, the equations of motion and hydrodynamic force estimates of both models were derived using the body fixed coordinate system shown in Figure 2(b) and made functions of the $\operatorname{cog}$ and cob locations so that when combined, they attempt to approximate the total system dynamics. Note that hydrodynamic effects are considered for each model individual, and their change when combined motion is not considered.

Models generated for both Starbug and Amour were separately written in such a way that they could be combined for cooperative control. Each vehicles equations of motion were derived using body fixed coordinates.

The equations of motion for each AUV were written in the form: 


$$
\begin{aligned}
\mathbf{F}_{b}+\mathbf{F}_{g}+\mathbf{F}_{c}+\mathbf{F}_{h} & =m \mathbf{a}_{c} \\
\mathbf{M}_{b}+\mathbf{M}_{g}+\mathbf{M}_{c}+\mathbf{M}_{h} & =\mathbf{I}_{c} \dot{\omega}+\omega \times \mathbf{I}_{c} \omega
\end{aligned}
$$

However, when the vehicles are combined Amour is physically locked onto Starbug and transfers forces from its thrusters to Starbug in the $x, y$ and $z$ directions, including moments about $x$ and $y$, whilst free to spin about the $z$-axis. The coordinate system is fixed to Starbug located at the combined vehicle's center of gravity. The total mass, weight and buoyancy of the combined system is the linear addition from both vehicles, and the combined inertia is determined using the parallel axis theorem. The combined center of gravity and buoyancy can are also easily calculated.

In this analysis, it is assumed that Amour will provide the control forces for the combined system. Due to the docking mechanism, any pure yaw moment applied to this vehicle results in a rotation about $z$ of Amour only, not Starbug. Therefore, an another equation was added to represent the yaw dynamics of Amour which is uncoupled from that of Starbug.

Estimation of hydrodynamic forces was performed by representing each vehicle as a series of $k$ cylinders and flat plates. Each hydrodynamic force and moment for each element is proportional to the square of the linear and angular velocities given by

$$
\begin{aligned}
& \mathbf{v}=(u, v, w) \\
& \omega=(p, q, r)
\end{aligned}
$$

The linear velocities at element $i$ located at $\mathbf{r}_{i}$ relative to the center of gravity is given by

$$
\mathbf{v}_{i}=\mathbf{v}+\omega \times \mathbf{r}_{i}
$$

However, if Amour spins relative to Starbug, the resulting yaw angle between the $x_{s}$ and $x_{a}$ axes $\left(\psi_{s / a}\right)$ in the $x y$ plane results in different velocities relative to Amours coordinate system. Therefore, the velocities need to be transformed to Amours

$$
\mathbf{T}=\left[\begin{array}{ccc}
\cos \left(\psi_{s / a}\right) & -\sin \left(\psi_{s / a}\right) & 0 \\
\sin \left(\psi_{s / a}\right) & \cos \left(\psi_{s / a}\right) & 0 \\
0 & 0 & 1
\end{array}\right]
$$


A similar approach is used for the angular velocities. Additionally, as the control forces act in Amours coordinate system, these too must be transformed to the combined coordinate system for evaluating the system dynamics.

For each AUV, it is assumed that the key mass, inertial and geometric properties are known. It is also assumed that their center of gravity lies on the $x z$ plane. As both AUV's have limited, if any, experimentally determined hydrodynamic coefficients, only low order hydrodynamics and added mass effects are included and approximated using geometry and estimates of drag and lift coefficients with each elements contribution is superimposed. This assumption typically leads to the greatest source of modelling error. Note that hydrodynamic effects are considered for each model individually, and their change when combined together is not considered. These models were individually tuned to approximate vehicle performance measured and observed during experimentation.

\section{Experiments and Results}

In a series of novel experiments Starbug has reliably located hundreds of Aquafleck nodes using the computer vision algorithms. The two AUVs docked with each other as shown in Figure 2 and performed cooperative control tasks. Amour located Starbug using the beaconbased location and docking algorithms. The cooperation tasks included both a rescue type mission whereby Amour docked onto Starbug and moved it around without any control input from Starbug, and another task in which Amour was slaved to control demands supplied by Starbug. The robots maneuvered together for over 2 hours.

Figure 3 shows the measured response of the combined vehicle's cooperative motion in which Starbug commanded Amour to yaw 130 degrees then move in its forward direction while Starbug used its onboard pressure sensor to provide vertical control commands to Amour for depth control. Note that no pitch or roll control was performed in this experiment (Note that at $t=14 \mathrm{~s}$, the vehicles reached the wall of the test tank, hence the large nose down pitch observed). Additionally, the figure shows the simulated motion provided by the combined model for comparison. The simulation model provides a reasonable estimate of the combined vehicle motion and its accuracy is considered suitable for developing further strategies for cooperative control and for anticipating the dynamics of system. 


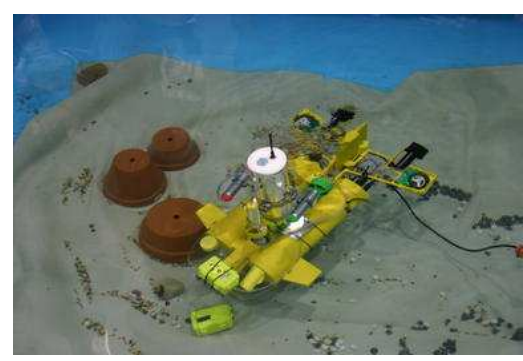

(a)

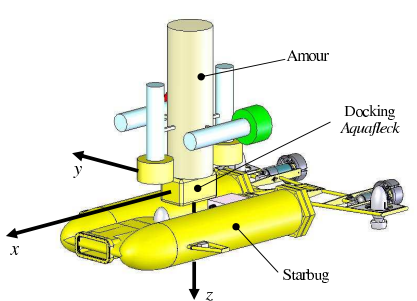

(b)

Figure 2: (a) AUVs during cooperative experiments. (b) Configuration and coordinate system.

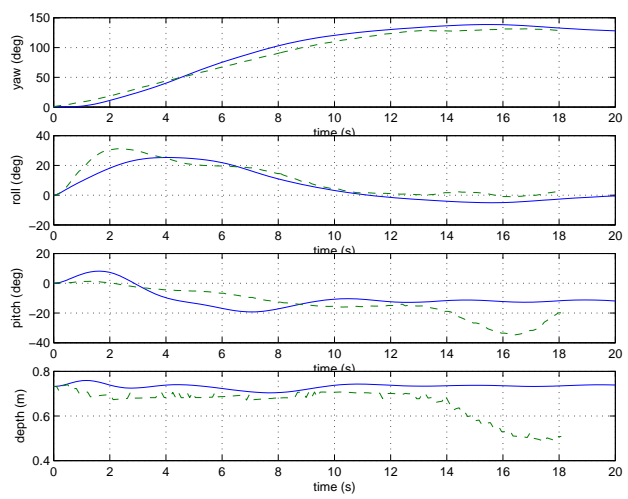

Figure 3: Comparison of simulated cooperative vehicle motion (solid line) against measured experimental results (dashed line).

Figure 4 shows results from cooperative control experiments with Starbug and Amour that demonstrate (1) closing of the loop with respect to position control and (2) action selection.

\section{Conclusions}

We have described an underwater robot and sensor network system that enables long-term underwater monitoring applications through cooperation. The very different AUVs are able to dock and navigate 

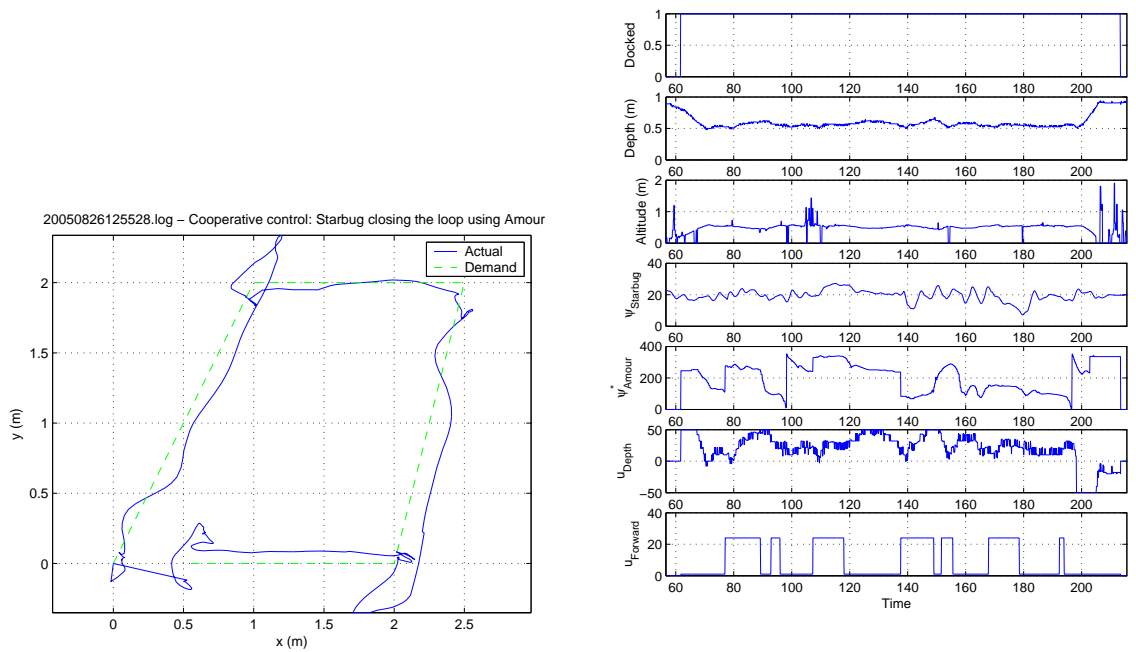

Figure 4: (Left) the path of the docked AUVs (as estimated by Starbug's vision system). Starbug sends thrust and yaw commands to Amour whist docked. (Right) data collected during a cooperation missing. Initially, Starbug is sitting on the bottom of the pool. Amour docks and lifts Starbug at $\mathrm{t}=62 \mathrm{~s}$. Following docking, Starbug provides Amour with control inputs to hold depth at $0.5 \mathrm{~m}$. The vision based altitude is shown as well. Starbug's goal was to hold a constant yaw angle of 20 degrees throughout the mission. Amour's yaw demand is shown to complete path along with the depth input and forward thrust command.

coupled together pulling resources using networked communication. We have described the control algorithm and experimental results obtained in the pool. In June 2006 we will test the control system in the ocean. We believe that cooperation is a key ingredient in creating underwater networked systems of robots and sensors capable of group motion and behavior and long-term operation. This paper presents some first steps in this direction.

\section{Acknowledgements}

We are grateful to NSF, Intel, and the ONR PLUSNet project for partial funding. We thank Keith Kotay and to the entire team at CSIRO for the technical and moral support provided during a period 
of intense experimentation; Pavan Sikka for the optical communication stack; Poe Chen for the interface between Flecks and Starbug and support for data muling; and Leslie Overs for electronics support.

\section{References}

[1] Autonomous Ocean Sampling Network (AOSN)II, collaborative project. http://www.princeton.edu/ $\tilde{\mathrm{d}} \mathrm{csl} / \operatorname{aosn} /$.

[2] Matthew Dunbabin, Jonathan Roberts, Kane Usher, Graeme Winstanley, and Peter Corke. A hybrid auv design for shallow water reef navigation. In Proceedings of the 2005 International Conference on Robotics and Automation, pages 2117-2122, Barcelona, April 2005.

[3] Matthew Dunbabin, Iuliu Vasilescu, Peter Corke, and Daniela Rus. Data muling over underwater wireless sensor networks using an autonomus underwater vehicle. In Proceedings of the 2006 International Conference on Robotics and Automation, page accepted, Orlando, FL, USA, May 2006.

[4] E. Fiorelli, N. E. Leonard, P. Bhatta, D. Paley, R. Bachmayer, and D. M. Fratantoni. Multi-AUV control and adaptive sampling in Monterey Bay. In Proceedings of the IEEE Autonomous Underwater Vehicles: Workshop on Multiple AUV Operations, Sebasco, ME, USA, June 2004.

[5] M. Nahon. A simplified dynamics model for autonomous underwater vehicles. In Proceedings of the Symposium on Autonomous Underwater Vehicle Technology (AUV'96), pages 373-379, 2-6 June 1996.

[6] I. Vasilescu, M. Dunbabin, P. Corke, K. Kotay, and D. Rus. Data collection, storage, and retrieval with an underwater sensor network. In Proceedings of the ACM Sensys 2005, San Diego, CA, USA, November 2005.

[7] Junku Yuh. Design and control of autonomous underwater robots: A survey. Autonomous Robots, 8(1):7-24, 2000. 